\title{
Adding Grey Level Information to Point Distribution Models Using Finite Elements
}

\author{
J.A. Marchant \\ Agricultural and Food Research Council \\ Silsoe Research Institute \\ Wrest Park, Silsoe, Beds. MK45 4HS
}

\begin{abstract}
Point distribution models are flexible shape models in which a shape is represented by a set of labelled points on the boundary. This paper uses techniques drawn from the Finite Element Method to include grey level information in such models. As both Point Distribution Models and the Finite Element Method represent objects using a set of labelled points, the techniques are very compatible. Results are presented which show that relatively complex objects, animal bodies, can be represented by a small number of elements. The method will eventually be used to improve the chances of successfully fitting Point Distribution Models to image data.
\end{abstract}

\section{Introduction}

The ability to monitor a visual scene containing animals and to draw intelligent conclusions automatically would have a significant impact on agricultural practice. For example, if the gait of an animal could be objectively measured, early detection of lameness would be possible. If the motion of a sow and piglets could be analysed, a stockman could be alerted if the piglets were not feeding properly.

One of the most basic tasks faced by any image analysis system is to segment the scene into animal and non-animal parts. This is made more difficult if the animals can touch each other or overlap.

The author has investigated the use of snakes to find animal boundaries ${ }^{[1]}$ even using neural network techniques to train the snake properties ${ }^{[2]}$. However, snakes contain no knowledge of the expected shape of the animals to be segmented whereas this type of knowledge can be a powerful aid to segmentation.

Unlike most manufactured objects, natural ones do not have a fixed shape and so any method of shape description should contain within it expected variations of shape. Recently, Cootes ${ }^{[3]}$ proposed a method for constructing flexible shape models in which a shape is represented by a set of labelled points on the boundary - 
a "Point Distribution Model". He also showed that the model could capture the major modes in which the shape could change by using Principle Component Analysis on the geometrical relationships between the labelled boundary points. This method gave a model for the boundary using only a few parameters to represent quite complex shapes. The models were derived by hand labelling a set of objects (the training set) at characteristic points on their boundaries and Cootes and others ${ }^{[4,5]}$ showed that the models could be used to search images for instances of the objects they represented.

As reported, the models represent boundary shapes only and are thus fitted to gradient data. This means that the model fitting procedure will be subject to noise and false minima associated with fitting boundaries to gradient images. More robust fitting may be achieved if the model also contained information on the way the grey level was likely to change over the surface of the object.

\section{A Method for Adding Grey Levels}

\subsection{Types of object to be represented}

Most of the animals that the author is currently concerned with have smoothly varying grey level distributions across patches of their surface. These patches can be of irregular shape and different sizes but the patch boundaries are reasonably smooth. Examples are the snout, ears, head, abdomen, etc. The method chosen has taken advantage of this restriction.

\section{$2.2 \quad$ Finite Elements}

Fig. 1 left, shows an animal component boundary (it is, in fact, the author's index finger) as it might have been labelled by Cootes. Fig. 1 right, shows the object relabelled using the new method. Note that there are a few internal points and the lighter lines represent internal patch boundaries. Note also that Cootes' method will work equally well on models which have internal points.

The principle behind the method reported here is to regard the patches as Finite Elements. The Finite Element Method (FEM) ${ }^{[6]}$ is a technique primarily used in stress analysis, but in fact can be used in many situations where partial differential equations need to be solved. The p.d.e. is cast into a variational form where the solution reduces to minimising an integral over a region subject to boundary conditions. The region is divided into a number of patches (the finite elements) in which the function to be minimised is represented as a low order polynomial. The value of the function at any point within an element is obtained by interpolating from the values at certain points on the element boundaries (the nodes). The aspect of the FEM used here is solely the interpolation of a function (the grey level) over a patch from points on the patch boundary. 

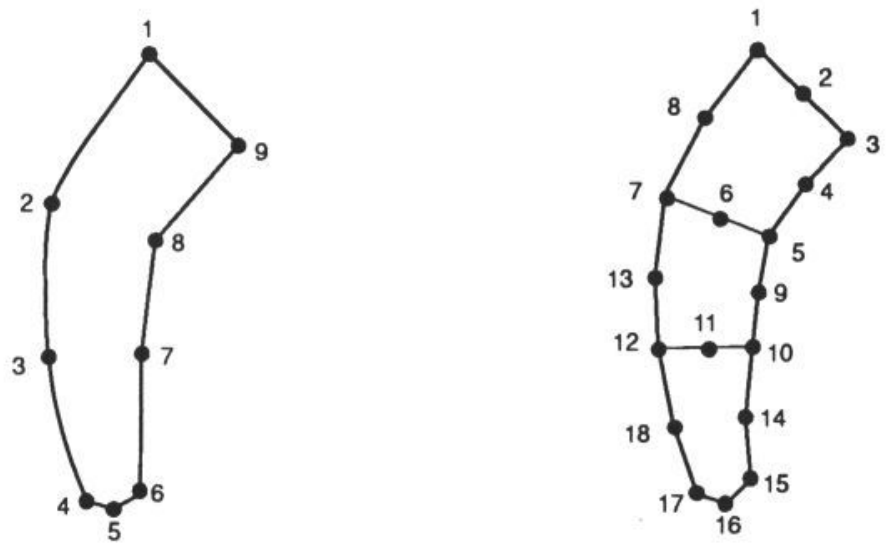

Fig. 1. Point distributions for an index finger. Left, points only on boundary. Right, set of points suitable for the Finite Element Method, and division into elements.

Various types of element are possible. Generally a balance is struck between element size (small enough to represent the changes in the function over the region) and the complexity of the interpolation (a higher order polynomial can represent the function better as the element size increases). The element used here starts as a square with a node at each corner and one at the mid-point of each side (Fig. 2, left). Of course, the use of square elements only would place such a severe restriction on the method as to render it useless. Hence a method is provided to map any curvilinear quadratic shape (e.g. Fig 2, right) into a square in a local coordinate system (Fig. 2, left). This is done with the matrix transformations

$$
\begin{aligned}
& x=[N][x]^{e} \\
& y=[N][y]^{e}
\end{aligned}
$$

where $[\mathrm{x}]^{\mathrm{e}}$ and $[\mathrm{y}]^{\mathrm{e}}$ are $8 \times 1$ matrices of the $\mathrm{x}$ and $\mathrm{y}$ node positions, and $[\mathrm{N}]$ is a matrix $(1 \times 8)$ of "shape functions" whose elements are functions of the local coordinates $\xi$ and $\eta$. Zienkiewicz ${ }^{[6]}$ tabulates shape functions for various elements. An elegant feature of some element classes (e.g. the one used here) is to use the same formulation to distort the shape and to interpolate the function within the element, i.e.

$$
\mathrm{g}=[\mathrm{N}][\mathrm{g}]^{\mathrm{e}}
$$

where $\mathrm{g}$ is the grey level at any point within an element and $[\mathrm{g}]^{\mathrm{e}}$ is an $8 \times 1$ matrix of grey levels at the nodes. 

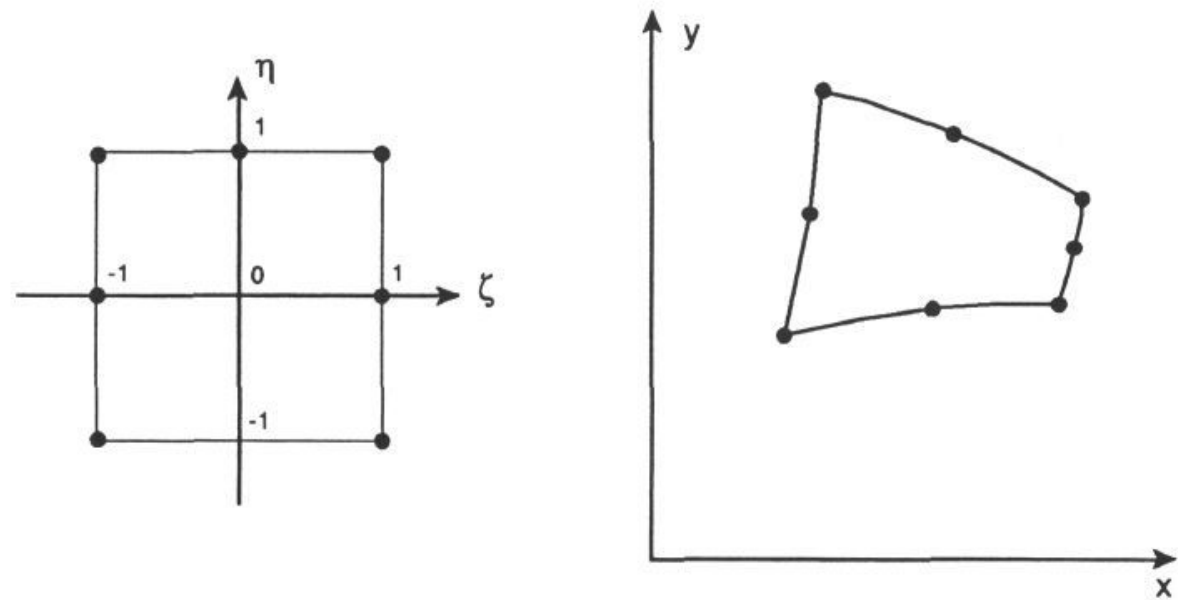

Fig. 2. Mapping of square element into distorted shape

To simplify the mathematics, calculations are done in the $\xi, \eta$ co-ordinate system. Thus, given the grey levels at the nodes of an element, the procedure for calculating the distribution over the rest of the element is to step through a grid of $\xi, \eta$ values and at each value:-

1. Calculate the x,y co-ordinates at the $\xi, \eta$ point using Eqns 1 and 2.

2. Calculate the grey level using Eqn. 3 .

The method outlined above can easily be coupled to Cootes' Point Distribution Models because both represent the object by using a collection of points and the FEM easily allows model distortion. Also both models are cast in matrix mathematics which eases the intellectual load on potential users. In addition, the FEM literature provides the ready made and well documented formulations necessary to implement the method.

\section{Some Results}

Fig. 3 shows four images of the finger example which is divided into elements, as in Fig. 1, right. The training set consisted of eight images with only one mode of variation, i.e. bending the finger. Fig. 4 shows four instances of the model generated using the method. The grey level values at the nodes were taken to be 


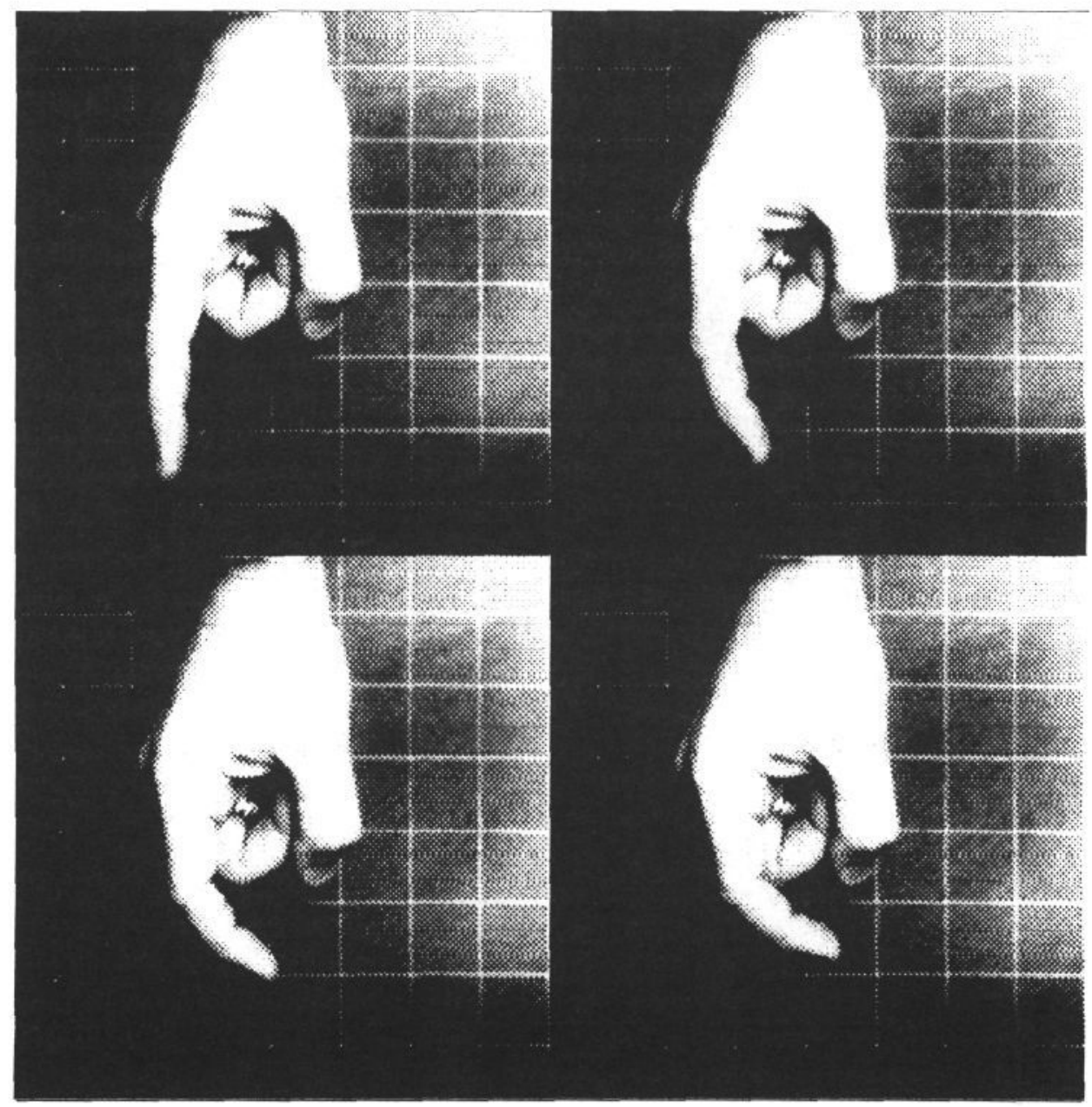

Fig. 3. Four images (from a sequence of eight) of the author's index finger 
an average of the grey levels over the corresponding points in each training image. Thus variations at each node are not taken into account here, although they could be. Note that the elements are successfully distorted to match the curvilinear shape of the actual boundary and the grey level varies across the model in a plausible way.

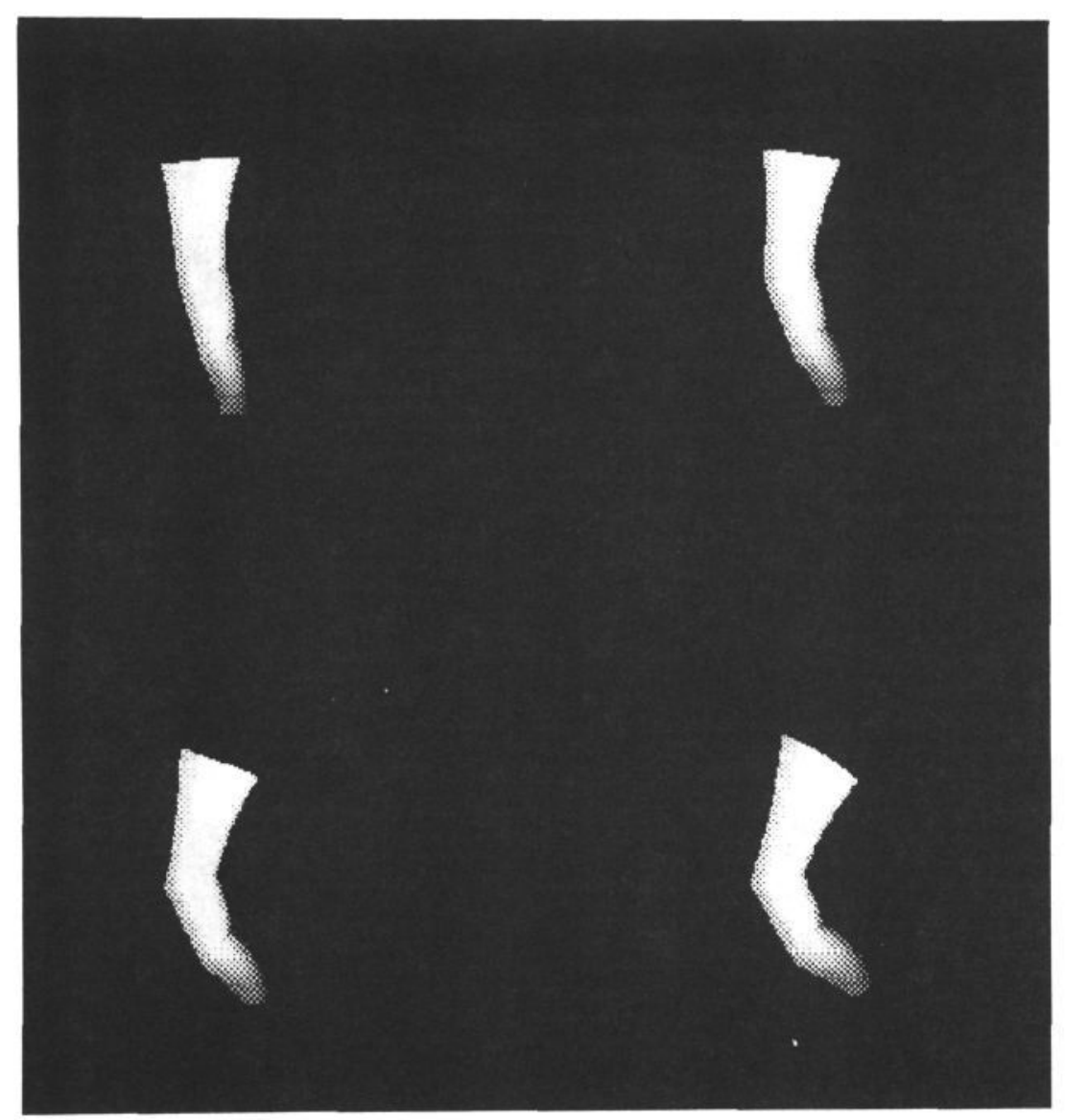

Fig. 4. Various instances of a model generated from the sequence in Fig. 3. 
Fig. 5 shows four plan view images (again from eight) of a pig moving below a camera. The pig is divided into the seven elements shown in Fig. 6. The elements have been chosen to represent reasonable physical divisions of the animal, over which the grey level changes in a smooth way. These are the snout, the two ears, the forehead, the head, the abdomen, and the rump.

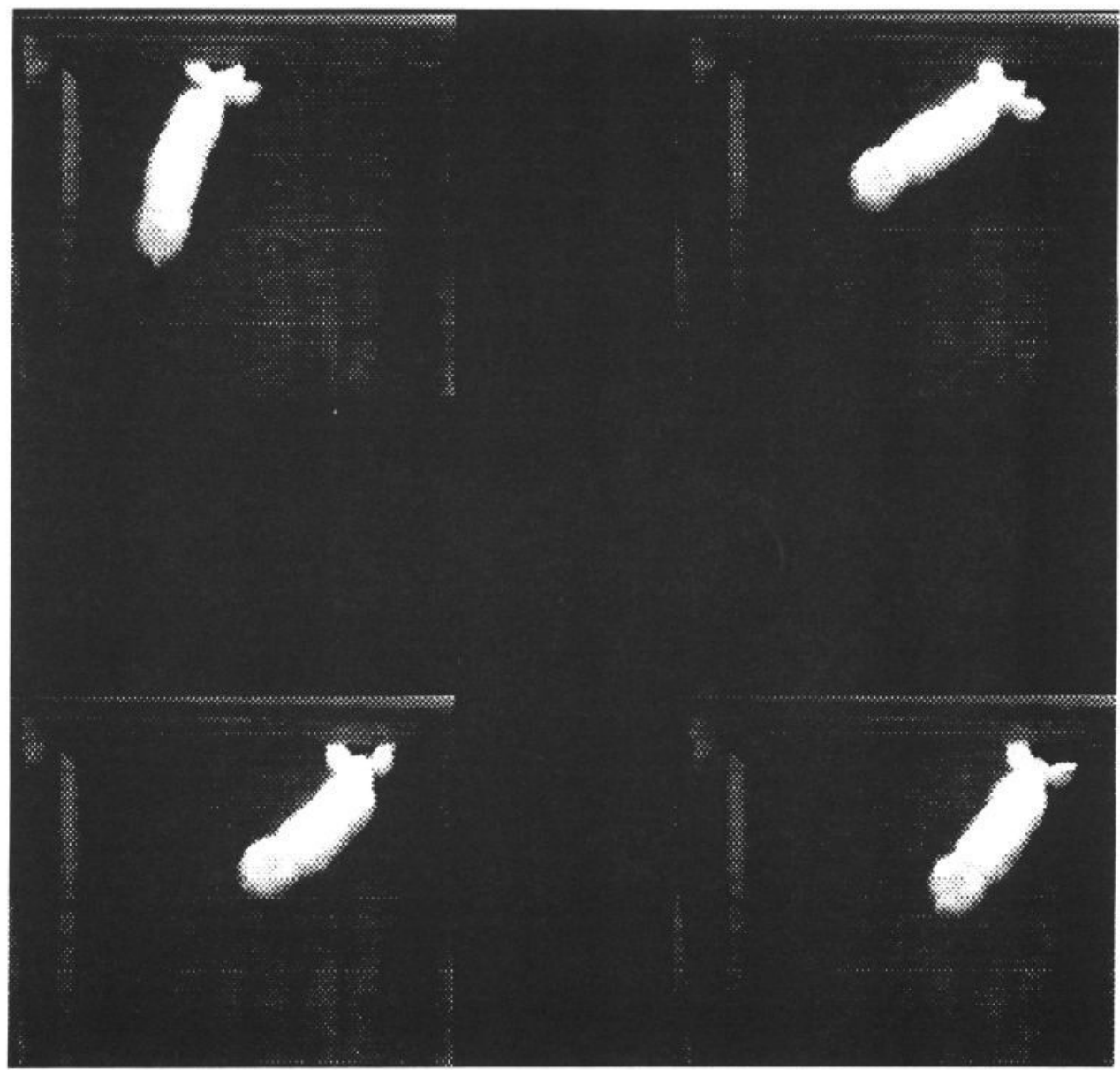

Fig. 5. Four images (from a sequence of eight) of a pig taken from above. 


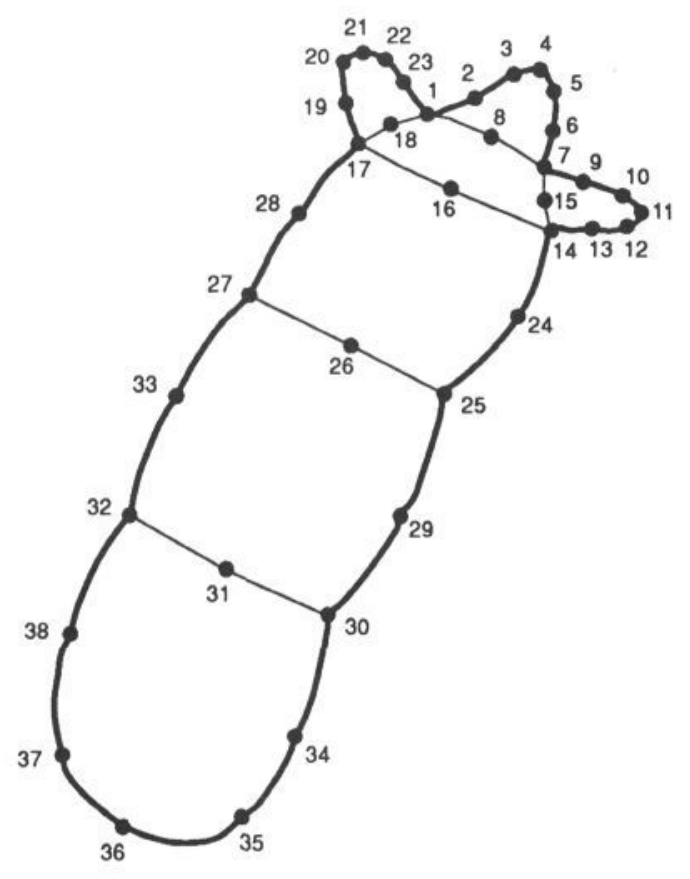

Fig. 6. Points and elements for a pig model.

During the image sequence the animal translated and rotated, bent its spine, walked and nodded its head. A video sequence shows the motion of the model very well, separate prints not so well. Unfortunately until printing technology allows moving sequences in papers we are stuck with the latter. However, Fig. 7 attempts to show three modes of variation. The top pair shows bending at one standard deviation each side of the mean, the middle the shuffling of the shoulders and rump at 1.5 standard deviations caused, presumably, by the walking action, and the bottom pair head nodding also at 1.5 standard deviations. Due to the restricted number of images in the training set, the bending action was only seen when accompanied by nodding (the animal was bending in order to pick up something from the ground). Hence the first mode regards the bending and nodding as inseparable. 

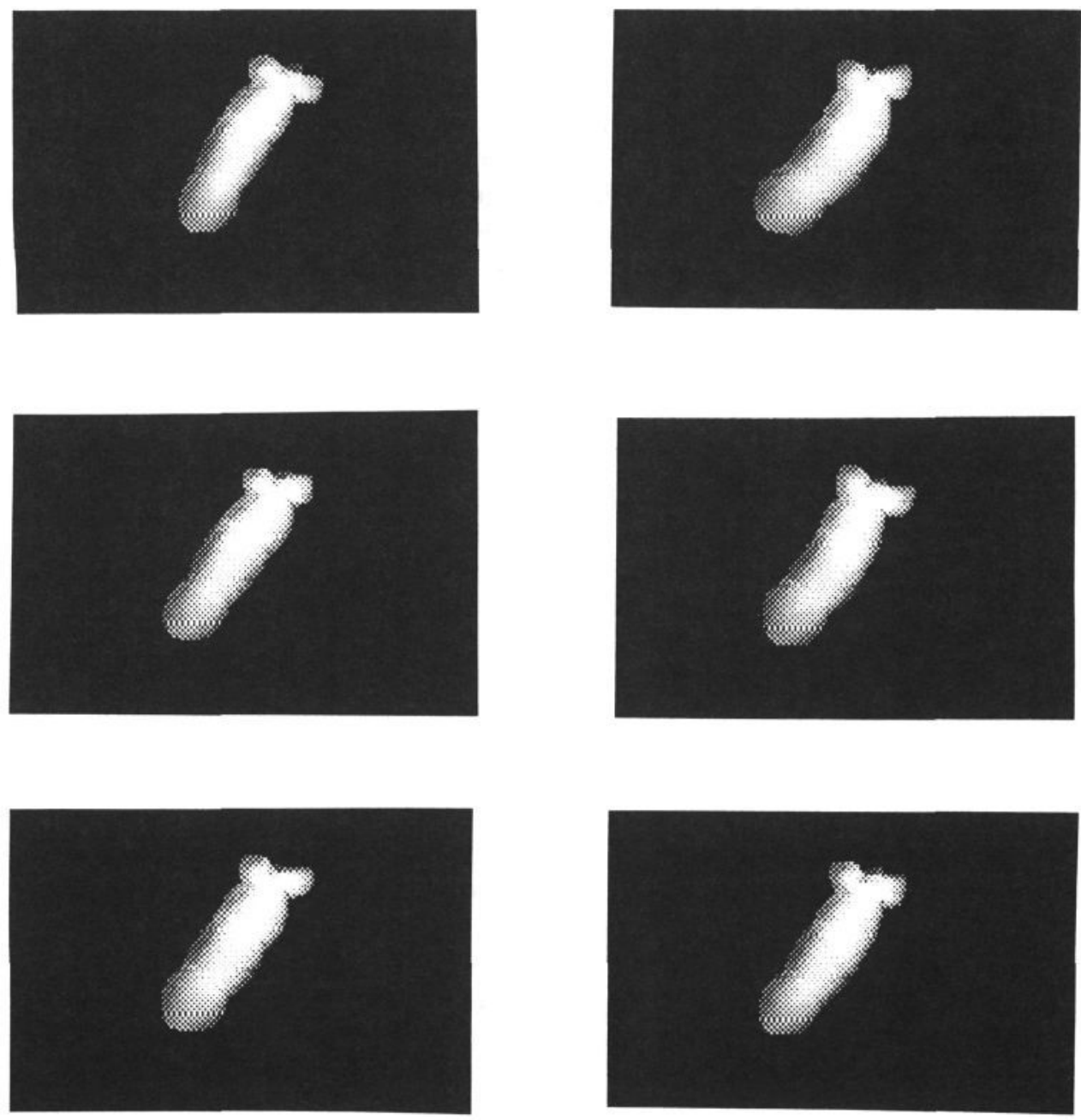

Fig. 7. Pig model generated from the sequence in Fig. 5 showing three modes of variation. Top, bending at 1.0 standard deviation from the mean. Middle, shuffling (walking). Bottom, head nodding. The last two modes at $\mathbf{1 . 5}$ standard deviations.

It can be seen that with only seven elements this fairly complex animal object has been reasonably well modelled both in terms of its boundary (Cootes' method) and its grey level (this companion method). The success depends on using a range of element sizes and shapes to suit the object's construction but this flexibility is a characteristic of the FEM. 


\section{Conclusions}

It is possible to use interpolation techniques drawn from the Finite Element Method to represent the grey level distribution over Point Distribution Models. With a relatively small number of elements, a plausible representation of complex objects can be generated. As both the FEM and PDM's are cast in terms of matrix algebra, both depend on representing the object by values at a set of points, and the FEM easily allows model distortion, the two techniques form a complementary whole.

It should be possible to use the models so generated to improve the chances of successfully fitting models to image data.

\section{References}

[1] J.A. Marchant and C.P. Schofield. Extending the snake image processing algorithm for outlining pigs in scenes. Computers and Electronics in Agriculture. (In press).

[2] P.F. Davis and J.A. Marchant. Pig image outlining using artificial neuron parameters in the snake contour method. Computers and Electronics in Agriculture (In press).

[3] T..F. Cootes, C.J. Taylor, D.H. Cooper, J. Graham. Training models of shape from sets of examples. Proc. British Machine Vision Conference, BMVA, Univ. of Leeds, 22-24 Sept. 1992., pp. 9-18.

[4] T.F. Cootes and C.J. Taylor. Active shape models - 'smart snakes'. Proc. British Machine Vision Conference, BMVA, Univ. of Leeds, 22-24 Sept. 1992, pp 266-275.

[5] A. Hill, T.F. Cootes, C.J. Taylor A generic system for image interpretation using flexible templates. Proc. British Machine Vision Conference, BMVA, Univ. of Leeds, 22-24 Sept. 1992, pp. 276-285.

[6] O.C. Zienkiewicz The finite element method (3rd ed.) McGraw-Hill, London, 1977. 